Artículo de investigación

Apuntes del CENES

ISSN 0120-3053

Volumen $34-\mathrm{N}^{\circ} .59$

enero - junio de 2015

Págs. 155-184

\title{
Papel de los seguidores en el desarrollo de las teorías de liderazgo organizacional
}

\author{
Role of the followers in the development of \\ theories of organizational leadership \\ Papeldos seguidoresno desenvolvimento \\ doteorias doliderança organizacional
}

Mónica García Solarte

Fecha de recepción: 20 de mayo de 2014

Concepto de evaluación: 15 de agosto de 2014

Fecha de aprobación: 28 de noviembre de 2014

\footnotetext{
Ingeniera Industrial. Magíster en Administración de Empresas de la Universidad del Valle, Master of Management of Tulane University, EE.UU. Profesora asociada de la Universidad del Valle, Facultad de Ciencias de la Administración, Departamento de Administración y Organizaciones, Sede San Fernando. Calle 4B No. 36-00 edificio 124 oficina 3013. Cali, Colombia. Correo electrónico: monica.garcia@correounivalle.edu.co
} 


\title{
Resumen
}

La mayoría de revisiones acerca del liderazgo se han enfocado en el líder y su papel dentro de la organización. Las investigaciones actuales demuestran que los seguidores, como un elemento importante del liderazgo, poseen un papel activo en la selección del estilo de liderazgo y en la teoría desde la cual se le evaluará a un líder. Desde este punto, el objetivo de este artículo es identificar cuál ha sido el papel de los seguidores en el desarrollo de las teorías del liderazgo organizacional. Al evaluar el papel que tiene el seguidor dentro del desarrollo de las teorías del liderazgo se pudo concluir que de acuerdo con su percepción, su comportamiento y sus necesidades, exige cierta categoría de líder, que puede ser vista desde cada teoría.

Palabras clave: seguidor, liderazgo, teorías del liderazgo, líder.

Clasificación JEL: M10, M50, M54.

\begin{abstract}
The most reviews about leadership have focused on the leader and his role in the organization. Modern research shows that the followers, as an important element of leadership, have an active role in the selection of leadership style and theory from which you evaluate a leader. From this point, the aim of this article is to identify what has been the role of followers in the development of theories of organizational leadership. In assessing the role of the follower in the development of theories of leadership, it was concluded that according to their perception, their behavior and their needs require a certain category of leader, which can be seen from each theory.
\end{abstract}

Keywords: follower, leadership, leadership theories, leader.

\section{Resumo}

A maioria dos comentários sobre a liderança centraram-se sobreo líder e papel dentro da organização. Pesquisas recentes mostram que os seguidores como um elemento importante de liderança, tem um papel ativo na seleção do estilo de liderança e teoria de que você será avaliado um líder. A partir deste ponto, o objetivo deste artigo é identificar o quetem sido o papel dos seguidores no desenvolvimento do teorias do liderança organizacional. Ao avaliaro papel doseguidor no desenvolvimento de teorias de liderança concluiu se que de acordo com sua percepção, seu comportamento e suas necessidades exigem ou exigir determinada categoria de líder que pode ser visto a partir de cada teoria.

Palavras-chave: teorias fan liderança de liderança, líder. 


\section{INTRODUCCIÓN}

El liderazgo se ha definido como la capacidad de influir en las personas para el logro de objetivos (Fleishman, Mumford, Zaccaro, Levin, Korotkin \& Hein, 1991). A lo largo del tiempo se le ha dado gran importancia al liderazgo dentro de las organizaciones, ya que con ello se puede lograr que las personas sean más productivas. Desde sus inicios, el liderazgo siempre ha estado centrado en el líder, en el estilo que este maneja y las teorías bajo las cuales este es reconocido.

No obstante, en las últimas décadas se ha empezado a reconocer la importancia que tiene el seguidor dentro el proceso del liderazgo. Can y Aktaş (2012), Carsten, Uhl-Bien, West, Patera y McGregor (2010), Collinson (2006) y Sy (2010), plantean la necesidad del estudio del rol del seguidor en el ejercicio del liderazgo y su importancia e influencia en el estilo del líder. En este sentido, el seguidor pasa a tener un rol tan relevante como el que tradicionalmente se le ha asignado al líder, por lo cual su proceso de influencia debe ser analizado, a fin de identificar y potencializar los procesos de interacción líder-seguidor y seguidor-líder que garantizanla efectividad del liderazgo en las organizaciones.

Muchas investigaciones como las de Gil, Alcover, Rico y Sánchez (2011), Bass (1985), Burns (1978), Conger y Kanungo (1998), Howell y Shamir (2005), House, Spangler y Woycke (1991), plantean la importancia del papel del seguidor pero no entran a profundizar en el papel que ejerce cuando es visto en el desarrollo de cada teoría. Por este motivo, el objetivo del presente artículo es identificarel papel de los seguidores dentro del desarrollo de las teorías del liderazgo organizacional. 
La teoría de los rasgos, del comportamiento, de contingencia y emergente, son las que se han seleccionado para la identificación de este papel activo de los seguidores.

Las conclusiones que arroja esta revisión identifican el papel activo de los seguidores en el proceso del liderazgo y la eficiencia en la organización. Así mismo, los seguidores, vistos desde la teoría de los rasgos y el comportamiento, poseen un papel un poco más pasivo enfocado en la identificación con el líder y en la percepción que este tiene de él. Desde la teoría de la contingencia, el papel que ejerce el seguidor es mucho más activo y empieza a identificarse más, ya que esta teoría plantea específicamente el estilo de liderazgo que se ejerce de acuerdo con ciertas características de los seguidores y el contexto en general. La teoría emergente, por su parte, plantea que el papel del seguidor está dado por las necesidades que este le plantea al líder para establecer una relación en la que ambos ganan.

En esta revisión, inicialmente, se defineel liderazgo; más adelante se plantean las teorías del liderazgo, la decisión de seguidor y el papel que ejerce; asimismo, se expone la relación líder y seguidor, y, en último término,se señala el papel del seguidor en el desarrollo de cada una de las teorías del liderazgo.

\section{LIDERAZGO}

Actualmente, las organizaciones tienen un nivel de exigencia más alto frente a la persona que se encuentra al mando. Los líderes se han convertido en una exigencia continua dentro de las organizaciones, ya que estos son los que, en términos administrativos, conducen allogro de la visión de la organización.

De manera más específica, se ha probado que el estilo de liderazgo influye sobre la conducta de los individuos en la empresa (Shamir, House \& Arthur, 1993), además, afecta los procesos de trabajo grupal, el clima social y los resultados (Evkall \& Ryhammar, 1997; Kahai \& Sosik, 1997). Rahman (2001) ha concluido que el estilo de liderazgo, los procesos, los productos y los servicios, así como las personas y el enfoque a los clientes, explican los resultados de las organizaciones.

El interés por esta temática se ha desarrollado desde el siglo XX (Nader, 2012). A pesar del interés por este fenómeno, Antonakis, Cianciolo y Sternberg, (2004) sostienen que gracias a la complejidad del fenómeno,la consolidación del concepto aún deja muchas preguntas sin responder.

Muchos teóricos han intentado plantear un concepto claro del liderazgo y han generalizado que este se refiere a la influencia que ejerce una persona sobre su grupo de referencia. A través de esta influencia, el líder logra que otros lleven a cabo las tareas que se pretenden desarrollar al servicio de la eficacia colectiva (Fleishman et al., 1991). El éxito de la organización es el principal criterio para medir la eficacia del liderazgo. Los líderes son tradicionalmente tratados dentro del 
marco de lo positivo y negativo, dependiendo de su desempeño en el interior de la organización (Kelley, 1988; Meindl, 1995).

Los líderes construyen la naturaleza de los problemas de la organización, desarrollan y evalúan las soluciones potenciales y planean e implementan las decisiones tendientes a resolver dichas problemáticas (Zaccaro, 1995). La base cognitiva y los valores de los líderes limitan el campo de la percepción selectiva, la interpretación de los problemas, la definición y valoración de las posibles soluciones y la selección e implementación de la estrategia. Por lo tanto, el estilo de liderazgo es una variable que tiene efectos directos sobre los procesos decisionales y sobre los resultados de las organizaciones (Waldman et al., 2001).

Lussier y Achua (2002, p. 6) se refieren al liderazgo como un: "proceso en el cual influyen líderes sobre seguidores y viceversa, para lograr los objetivos de una organización a través del cambio". En este sentido, estos autores señalan que el liderazgo tiene una serie de elementos que deben ser considerados, tales como: influencia, líderes y seguidores, personas, cambio y objetivos organizacionales.

Theime y Treviño (2012, p. 40) se refieren al liderazgo como la influencia que el comportamiento del líder puede tener en el desempeño del grupo: "Los diversos estudios sobre el liderazgo explicado en términos de comportamientos tuvieron un éxito modesto al tratar de identificar relaciones consistentes entre patrones de comportamiento del líder y el desempeño del grupo". Esta explicación se debe al estudio del comportamiento que en un momento no contemplaba el elemento situacional para el ejercicio del liderazgo;sin embargo, se acepta el hecho de que la probabilidad del éxito del liderazgo es un tema complejo que no solo depende de una serie de comportamientos (Theime \& Treviño, 2012).

Asimismo, Northouse (2001), dentro de la definición de liderazgo, rescata cuatro componentes:

a) Es un proceso, no una característica que reside en el líder, no es algo lineal, más bien es un evento interactivo el cual ocurre entre el líder y los seguidores.

b) Involucra influencia del líder hacia los seguidores reales y potenciales.

c) Ocurre en grupos que son el contexto donde el liderazgo tiene lugar.

d) Incluye atención a las metas que se da al grupo de individuos.

Aunque el término se ha conceptualizado de diferentes formas, existe cierto acuerdo en asociarlo a percepciones y atribuciones de los seguidores, influenciados por las cualidades y comportamientos del líder, las situaciones de contexto, y por las necesidades individuales y colectivas de los seguidores (Yuk1, 1998). En este sentido, Padilla y Quintana (2012) proponen que otra forma de analizar el liderazgo es a partir de su construcción teniendo en cuenta quien es liderado y no quien lidera. 


\section{TEORÍAS DEL LIDERAZGO}

El liderazgo es uno de los temas ampliamente tratados en el campo de las organizaciones. Por ejemplo, la identidad del líder, la forma en la que el líder conduce, la respuesta de los subordinados, la relación líder-seguidor, son algunos de los temas que se tratan en varios estudios. En este contexto, las primeras teorías sobre liderazgo enfatizaron en las características del líder, determinadas más bien genéticamente que socialmente, y dichas características pasaron a ser el determinante de las habilidades del líder. Posteriormente surgió la teoría de contingencia del liderazgo, en la cual los factores situacionales y la personalidad del líder eran los aspectos esenciales para delinear la relación líder-seguidor. Otras teorías focalizaron su interés en los mecanismos de decisión y en las situaciones organizacionales, o en el balance entre enfoque a la tarea y a las relaciones humanas. Sin embargo, la concepción de liderazgo transformacional y liderazgo transaccional ha recibido la mayor atención en los últimos años, y está dentro de las teorías emergentes.

\section{Teoría de los rasgos}

La teoría de los rasgos se centró en identificar las características personales que dan lugar al líder. Bajo el criterio de esta teoría se pensaba que los líderes efectivos deben tener ciertas características o cualidades personales que están por encima de las demás personas. Desde los estudios de Fayol (1986), se ha planteado que solo ciertos rasgos personales suelen estar asociados al liderazgo, sin embargo, este autor no lo expone directamente, sino que destaca sus estudios de rasgos enfocados a la descripción de capacidad técnica y capacidad administrativa.

Para Fayol (1986), los rasgos que deben reunir los grandes jefes son salud y vigor físico, inteligencia y vigor intelectual, cualidades morales: voluntad reflexiva, firme, perseverante; actividad, energía $\mathrm{y}$, si hay lugar a ello, audacia; valor para las responsabilidades; sentimiento del deber; preocupación por el interés general, cultura general, conocimientos administrativos, nociones generales referentes a las otras funciones y la más amplía competencia posible en la profesión especial característica de la empresa.

Dentro de los rasgos más comunes se encuentran la inteligencia, conocimiento y experiencia, dominio, confianza en sí mismo, alta energía, tolerancia al estrés, integridad y honestidad, y madurez (Jones \& George, 2010). Aunque esta teoría se ha enmarcado dentro de los rasgos, no necesariamente todos están sujetos a la personalidad. Muchos de los rasgos están sujetos a las aptitudes, habilidades, conocimientos y experiencias que posee el individuo.

\section{Teoría del comportamiento}

Esta teoría fue planteada en la Universidad Estatal de Ohio, donde se estudiaron los diferentes comportamientos que tienen los individuos que son líderes. 
Lussier y Achua (2002) señalan que esta teoría propone el reconocimiento de que en las organizaciones no solo se necesitan resultados sino también personas. La idea de estos dos autores es que la teoría del comportamiento se basa en reconocer los estilos de liderazgo de acuerdo con su labor.

A partir de este nuevo enfoque, la preocupación no está concentrada en los rasgos del líder, sino en lo que hace y como lo hace (Palomino, 2009). Este enfoque contribuyó a que diferentes autores se enfocaran en identificar los patrones de un individuo que hacen que pueda influir en un grupo o en una organización (Robbins, 1999).

En sus inicios, Likert (1961) propone que la administración es un proceso que asume posiciones diferentes en cada organización, dependiendo de las condiciones internas y externas de esta. A partir de ello, sugiere características propias de las organizaciones que configuran estilos de liderazgo. Según la forma en que estas características se presentan en los administradores, se pueden identificar cuatro sistemas o estilos de dirección (Liker 1961): sistema 1. Administración explosiva o autoritaria; sistema 2. Administración benevolente-autoritaria; sistema 3 . Administración consultiva; y sistema 4. Administración de grupo participativo.

Lewin (1951) dio una de las primeras explicaciones de los estilos de liderazgo, con el argumento de que estos surgen del uso que los dirigentes dan a la autoridad que poseen. A partir de esto, le fue posible establecer tres estilos de dirección o liderazgo que obedecen a su criterio de análisis: el estilo de liderazgo autocrático, democrático y laissez-faire. Estos estilos de liderazgo están basados específicamente en el comportamiento del líder.

Finalmente, Blake y Mouton (1964) desarrollaron una de las herramientas más conocidas en lo que se refiere a la medición o definición de los estilos de dirección, el grid gerencial, que fue actualizada con el apoyo de otros colegas y ha sido adaptada a diversas áreas de la administración.La matriz fue desarrollada en el marco de dos dimensiones: el interés por la producción (resultados) y el interés por las personas. Su importancia radica en indicar el carácter o la fuerza de los supuestos y los valores en que descansa cualquier enfoque (Blake \& Mouton, 1964). Esta herramienta permite identificar hechos, opiniones, actitudes y emociones a partir de una visión introspectiva. En este sentido, estos autores hicieron un gran aporte a la teoría del comportamiento.

\section{Teoría de la contingencia}

El poseer ciertos rasgos o comportamientos no garantiza la existencia del líder. En este aspecto, esta teoría rompe con los esquemas y plantea que todas las situaciones requieren de liderazgo. El líder está expuesto a un contexto en donde se desarrolla con sus seguidores, por lo tanto, esa variedad de situaciones hace que la teoría de contingencia o situacional surja como un liderazgo eficaz dependiendo de 
la situación. Esta teoría está sustentada por las investigaciones realizadas por Fiedler (1967), Vroom y Yetton (1973), Evans (1970) y House (1971), entre otros. En este enfoque, los autores plantean que quien quiera ejercer como líder debe ser capaz de captar con rapidez las peculiaridades de las diversas situaciones con que se encuentre y seleccionar para cada una de ellas el estilo de liderazgo más apropiado (Palomino, 2009).

En el marco de esta teoría no existe un solo tipo de liderazgo, sino un estilo que se adecue a cada una de las situaciones y opere en el contexto para ser efectivo (Palomino, 2009). Entre los factores que están relacionados y que influyen desde la perspectiva de esta teoría, según los plantea Guillen (2006), están la formación del seguidor, las necesidades de autorrealización del seguidor, la experiencia y la madurez. Por lo tanto, en este enfoque se empieza a considerar la relación existente entre el líder y el seguidor, desde la perspectiva en la que ambos influyen en el estilo de liderazgo (Palomino, 2009).

Un aporte fundamental para esta teoría fueron los modelos deFiedler (1967), Hersey y Blanchard (1969). Fiedler fue uno de los primeros investigadores de liderazgo en sostener que un líder debe ser contingente, es decir, que depende de las características del líder y la situación. En este modelo se señala que muchos individuos son líderes en una situación y no tanto en otras. Fiedler (1967) sostiene, además, que se debe considerar el estilo del líder para referirse a las característi- cas personales del individuo, y destaca principalmente al líder orientado a las relaciones y al orientado a las tareas. Los líderes orientados a las relaciones se interesan principalmente en tener buenas relaciones con sus subordinados y serle agradable, y los líderes orientados a las tareas se interesan especialmente en que los subordinados tengan niveles de desempeño altos y se enfoquen en el cumplimiento de la tarea (Jones \& George, 2010).

Fiedler también consideró las características situacionales para la identificación del líder. Indicó tres características situacionales determinantes de lo favorable de la situación para su dirección: relaciones líder-miembro, como la medida en que a los seguidores les agrada su líder, confían en él y son leales; estructura de las tareas, como medida en que las tareas a realizar están claramente señaladas y descritas; y poder del puesto, como la cantidad de poder legítimo, de recompensa o coercitivo que tiene el líder en virtud del cargo que ocupa.

Por otra parte, en el trabajo realizado por Hersey y Blanchard (1969) se señala que el liderazgo basado en el comportamiento está relacionado con las tareas y las relaciones, dependiendo de la relación que tenga con el seguidor.El aspecto innovador en las investigaciones de estos autores fue la presentación de los cuatro cuadrantes que simbolizan las dimensiones de tareas y relaciones, y las dimensiones de comportamiento que mencionan son: dirigir, persuadir, participar y delegar. 


\section{Teoría emergente}

Esta teoría se orienta principalmente al liderazgo transformacional. Este estilo de liderazgo se da cuando los líderes transforman a sus seguidores. En este sentido, este estilo propone que se realiza a través del logro de los seguidores en la medida que estos están plenamente conscientes de la importancia que sus puestos tienen para la organización. Asimismo, los líderes transformacionales logran que los seguidores estén conscientes de las necesidades personales de crecimiento, desarrollo y realización. Estos líderes motivan a los seguidores a que trabajen bien, no solo para el beneficio de la organización sino también para el beneficio individual (Jones \& George, 2010).

El liderazgo transformacional se genera cuando los líderes y seguidores se elevan unos a otros a los niveles más altos de los valores y las motivaciones (Burns, 1978). "El resultado de la transformación de liderazgo es una relación de estímulo recíproco y la elevación que convierte seguidores en líderes y puede convertir líderes en agentes morales" (1978, p.24).

Bass (1985) define un líder transformacional como el que motiva a los seguidores a hacer más de lo que se esperaba que hicieran. El punto fundamental de Bass es que los líderes transformacionales amplían y cambian los intereses de sus seguidores, al mismo tiempo que generan conocimiento y aceptación de los objetivos y la misión del grupo. Según este autor, el liderazgo transformacional consiste en cuatro factores: el carisma o influencia idealizada, el liderazgo de inspiración o motivación, la estimulación intelectual y la consideración individual.

Asimismo, Bass (1981) trabajó en una versión sobre el liderazgo transformacional basado en el trabajo realizado por Burns, House y otros autores, cuya propuesta se enfocó en las necesidades de los seguidores, más que en las del líder, y sugirió que el liderazgo transformacional puede aplicarse a situaciones en las cuales los resultados no son positivos.

\section{SEGUIDOR}

Para Lussier y Achua (2002), un seguidor es alguien que recibe la influencia de un líder, y puede ser administrado o subordinado, a la vez que brinda la categoría de reconocimiento del líder. Para Chaleff (1995), un seguidor no es un sinónimo de subordinado. Un subordinado se reporta a un individuo de mayor rango y puede, en la práctica, ser un defensor, un antagonista o un indiferente. El seguidor comparte un propósito común con el líder, cree en lo que la organización está tratando de llevar a cabo y quiere que tanto el líder como la organización triunfen.

A continuación, en la Tabla 1 se plantean algunos enfoques con respecto a la definición del seguidor y su papel en el ejercicio del liderazgo: 
Tabla 1. Los seguidores y su papel en el ejercicio del liderazgo

\begin{tabular}{|c|c|}
\hline Autor & Aproximación teórica \\
\hline $\begin{array}{l}\text { Kohles, Blighy } \\
\text { \& Carsten } \\
\text { (2012) }\end{array}$ & $\begin{array}{l}\text { Cuando los seguidores comparten e interiorizan los valores de una organización, } \\
\text { su deseo de permanecer en esta probablemente aumenta. Además, los procesos de } \\
\text { comunicación líder-seguidor deben ser bidireccionales y encontrarse relacionados } \\
\text { con la visión de la organización, ya que esto puede diferenciar a esta organización } \\
\text { de aquellas donde sus postulados se quedan solo en el papel. } \\
\text { Cuando una visión se centra claramente en los seguidores, al estar dirigida hacia } \\
\text { ellos y animarles a reflexionar sobre su contribución a la misma, los seguidores son } \\
\text { más propensos a crear un concepto de sí mismos asociados a un mayor rendimiento } \\
\text { y alineación con la organización. }\end{array}$ \\
\hline $\begin{array}{c}\text { Can \& Akta } \\
\text { (2012) }\end{array}$ & $\begin{array}{l}\text { Aunque en mucha literatura se hace énfasis en el papel central del líder dentro } \\
\text { del proceso de liderazgo y la influencia que este tiene en los seguidores, algunos } \\
\text { análisis llegan a determinar la importancia que los seguidores tienen por su parti- } \\
\text { cipación en la toma de decisiones. Los seguidores, según algunos estudios hechos } \\
\text { por Kelley (1988), muestran unas dimensiones del comportamiento que determinan } \\
\text { su papel y tipo de participación en el ejercicio del liderazgo, llevándolos a ser de } \\
\text { pensamiento crítico, innovadores, creativos o ejemplares. } \\
\text { Los tipos de seguidores son: alienado, ejemplar, pasivo y conformista. }\end{array}$ \\
\hline $\begin{array}{l}\text { Carsten, Bien, } \\
\text { West, Patera \& } \\
\text { McGregor } \\
\text { (2010) }\end{array}$ & $\begin{array}{l}\text { Señalan que algunos autores como Meindi (1995) han explorado como los segui- } \\
\text { dores construyen liderazgo; otros, como Felfe y Schyns (2006), analizan de qué } \\
\text { manera las características personales de los seguidores influyen en las percepcio- } \\
\text { nes de liderazgo. Sin embargo, se debe analizar el verdadero aporte del seguidor } \\
\text { al liderazgo y la forma como este construye una serie de interpretaciones que lo } \\
\text { llevan a establecer un rol significativo en las organizaciones. }\end{array}$ \\
\hline $\begin{array}{l}\text { Miller, Butler \& } \\
\text { Cosentino } \\
\quad(2003)\end{array}$ & $\begin{array}{l}\text { Los seguidores y su comportamiento se ven determinados por el grado de control } \\
\text { que el líder logre ejercer, por lo cual se requieren algunos elementos en el ámbito } \\
\text { de la interacción que faciliten una influencia precisa y adecuada por parte del líder } \\
\text { para garantizar la efectividad de los seguidores en sus funciones. }\end{array}$ \\
\hline $\begin{array}{c}\text { Collinson } \\
(2006)\end{array}$ & $\begin{array}{l}\text { Los seguidores nunca han sido más importantes que en la actualidad, cuando } \\
\text { diferentes autores han tratado de identificar el papel que estos desempeñan en el } \\
\text { liderazgo. Este papel va mucho más allá de una categorización de los seguidores } \\
\text { bajo ciertos estereotipos, pues el liderazgo actual es compartido y debe tener en } \\
\text { cuenta las capacidades, facultades y conocimientos de los seguidores. }\end{array}$ \\
\hline Sy (2010) & $\begin{array}{l}\text { Existe una serie de teorías implícitas del liderazgo que permiten identificar y expli- } \\
\text { car las relaciones líder-seguidor, teniendo como punto de partida el hecho de que } \\
\text { cada uno busca orientar sus comportamientos y acciones de acuerdo con el logro de } \\
\text { sus objetivos. Por lo cual, el rendimiento de los seguidores puede depender de su } \\
\text { percepción acerca del líder y de las interacciones que establezcan con los mismos. }\end{array}$ \\
\hline $\begin{array}{c}\text { Peterson \& } \\
\text { Avolio (2012) }\end{array}$ & $\begin{array}{l}\text { Los seguidores atribuyen características a aquellos líderes que consideran auténticos } \\
\text { y determinan el desempeño propio de acuerdo con el clima laboral que el líder } \\
\text { propicie para el desarrollo de las actividades. Por eso, cuando el seguidor percibe } \\
\text { intenciones positivas del líder, manifiesta un aumento en su desempeño, lo que } \\
\text { permite asumir que las emociones positivas o negativas de los seguidores tienen } \\
\text { relación directa con el desempeño individual y el logro de los objetivos. }\end{array}$ \\
\hline
\end{tabular}




\begin{tabular}{|c|c|}
\hline Autor & Aproximación teórica \\
\hline $\begin{array}{l}\text { Hoption, Christie } \\
\& \text { Barling (2012) }\end{array}$ & $\begin{array}{l}\text { El desempeño de un seguidor se puede ver afectado por la concepción que se } \\
\text { tenga con respecto al término que lo etiqueta. Esta concepción puede ser negativa } \\
\text { y orientada a pensar que el seguidor es un individuo sin ambiciones y totalmente } \\
\text { determinado por la influencia del seguidor, o por el contrario, puede ser visto como } \\
\text { un complemento para el ejercicio del liderazgo al determinar en cierta medida el } \\
\text { desempeño del líder. Sin embargo, en un proceso de liderazgo efectivo se debe } \\
\text { dar cabida a una concepción positiva del seguidor que asegure un efecto positivo } \\
\text { en el desempeño. }\end{array}$ \\
\hline $\begin{array}{l}\text { Sy \& Choi } \\
\text { (2013) }\end{array}$ & $\begin{array}{l}\text { Los seguidores pueden ser vistos como receptores del estado de ánimo del líder, } \\
\text { y determinan sus procesos de comportamiento y desempeño según la interacción } \\
\text { que este les facilite. Se espera que el seguidor propague este estado de ánimo que } \\
\text { el líder genera, en pro de un mayor éxito en el desarrollo de las labores. }\end{array}$ \\
\hline $\begin{array}{l}\text { Davidovitz, } \\
\text { Mikulincer, } \\
\text { Shaver, Izak \& } \\
\text { Popper (2007) }\end{array}$ & $\begin{array}{l}\text { La relación seguidor-líder puede explicarse desde la teoría del apego, tratando de } \\
\text { determinar los procesos de proximidad entre ambos, el objeto de la relación, la } \\
\text { toma de riesgos y el autodesarrollo. La inseguridad de los seguidores en cuanto a } \\
\text { su desempeño, afecta la relación líder-seguidor generando barreras psicológicas } \\
\text { y perturbando el rendimiento, el desarrollo y el ajuste al equipo. Sin embargo, } \\
\text { esta inseguridad se puede dar por la falta de empoderamiento, la cual es respon- } \\
\text { sabilidad del líder. }\end{array}$ \\
\hline $\begin{array}{l}\text { Howelly \& Hall- } \\
\text { Merenda (1999) }\end{array}$ & $\begin{array}{l}\text { La labor y efectividad del seguidor está determinada por los sistemas de recom- } \\
\text { pensas que negocia con el líder como consecuencia de su desempeño. En general, } \\
\text { las funciones, comportamientos y resultados del seguidor están definidos por el } \\
\text { líder y el tipo de liderazgo que decida implementar para el logro de los objetivos. } \\
\text { Se identifica una relación casi unilateral líder-seguidor. } \\
\text { En el intercambio líder-miembro y un management positivo, estos elementos } \\
\text { pueden predecir el desempeño del seguidor. Se espera que el líder implemente un } \\
\text { liderazgo transformacional que permita la obtención de mejores resultados por } \\
\text { parte de los seguidores. }\end{array}$ \\
\hline $\begin{array}{l}\text { Podsakoff, Mac- } \\
\text { kenzie, Moorman } \\
\text { \& Fetter (1990) } \\
\text { Lee, Almaza, }\end{array}$ & $\begin{array}{l}\text { Los seguidores vistos como el resultado de la influencia del líder. Los líderes } \\
\text { transforman los valores básicos, las creencias y las actitudes de los seguidores para } \\
\text { que estén dispuestos a llevar a cabo más allá de los niveles mínimos estipulados } \\
\text { por la organización. }\end{array}$ \\
\hline $\begin{array}{l}\text { Jang, Nelson \& } \\
\text { Ghiselli, (2013) }\end{array}$ & $\begin{array}{l}\text { Los seguidores vistos como dependientes del tipo de liderazgo que se ejerce, } \\
\text { particularmente del transformacional, que no influye de manera directa en ellos } \\
\text { pero sí en el clima organizacional, que, a su vez, tiene un cierto impacto en las } \\
\text { actitudes de los seguidores dentro del ejercicio del liderazgo. }\end{array}$ \\
\hline $\begin{array}{l}\text { Johnson, Lepine } \\
\text { \& Halverson } \\
\quad(2008) \text {, } \\
\text { Conger, Kanungo } \\
\text { \& Menon, (2000) } \\
\text { Palomino, Espi- } \\
\end{array}$ & $\begin{array}{l}\text { Los seguidores determinados por el carisma del líder, el cual estáasociado con afectos } \\
\text { positivos y negativos de los seguidores. Un líder carismático tiene seguidores que } \\
\text { son más positivos y orientados a la tarea, mientras que los seguidores de un líder no } \\
\text { carismático, tienen un desempeño bajo y no se orientan a la tarea. } \\
\text { Los seguidores influenciados por líderes carismáticos tienen mayor confianza, satis- } \\
\text { facción, sentido de identidad, mayor desempeño grupal y se sienten más empoderados. }\end{array}$ \\
\hline $\begin{array}{l}\text { noza \& Aguilar } \\
\text { (2013) citando a } \\
\text { Bass }(1990)\end{array}$ & $\begin{array}{l}\text { "Los seguidores tienen una mayor disposición a seguir a los líderes y son afectados } \\
\text { de forma diferente dependiendo del estilo de liderazgo en tiempo de incertidumbre. } \\
\text { Adicionalmente los seguidores tienden a sobreestimar la responsabilidad de los } \\
\text { líderes en los resultados de la organización bajo condiciones de incertidumbre". } \\
\text { Los seguidores necesitan sentir que son protegidos y valorados en la organización. }\end{array}$ \\
\hline
\end{tabular}

Fuente: elaboración de la autora 
Tal como se plantea en la Tabla 1 , muchos autores enfocan su análisis del papel de los seguidores en el liderazgo, a agentes pasivos determinados casi en su totalidad por las percepciones, emociones y procesos de influencia del líder. El comportamiento del seguidor y su desempeño dependen del grado en el cual el líder establezca procesos de interacción y de influencia efectivos. Sin embargo, autores como Sy (2010), Collinson (2006), Carsten, Uhl-Bien, West, Patera y McGregor (2010), Can y Aktaş (2012) proponen no solo la necesidad de considerar al seguidor sino su importancia en el ejercicio del liderazgo, situándolo en posición de privilegio al otorgarle la responsabilidad de influir en los actos del líder y el estilo que este decide adoptar.

Para Kelley (1992), los seguidores son aquellos empleados que son ejemplares, que hacen prosperar a las empresas. Davis y Newstrom (2000) mencionan que algunas veces los líderes también se convierten en seguidores, y que es una relación de subordinación dinámica, donde se muestra la capacidad de seguir. Por lo tanto, un seguidor es la persona que recibe influencia del líder y que lo sigue por convicción propia, que tiene un propósito común o cree en el propósito del líder y lo apoya en la consecución de las metas propuestas con entusiasmo y energía y que, a su vez, influencia al líder positivamente.

Sin embargo, existe cierto tipo de seguidores con los cuales se puede llegar a establecer contacto, a los que Kelley
(1992) clasifica en seguidores buenos, críticos, conformistas, pragmáticos y pasivos. Cada estilo de seguidor tiene un cierto nivel de participación y de ejercicio intelectual que determina su importancia y rol en el ejercicio del liderazgo, por lo tanto, cuanto mayor sea su relevancia, mayor será la exigencia que se realiza en estos sentidos.

Chaleff (1995) manifiesta que existen tres características principales que necesita el seguidor para desarrollar una buena relación con su líder. Estas son:

- Compromiso: la relación entre líderes y seguidores exige un compromiso mutuo. Compromiso significa lealtad mutua y confianza entre la gente, sus líderes y la organización. El compromiso es definitivamente una ventaja competitiva, pero no es gratuita. Los líderes deben ganarse el compromiso de sus seguidores. Líderes y seguidores que se hallan continuamente intercambiando puntos de vista, necesitan encontrar un lugar común para la lealtad que trasciende la inestabilidad de sus relaciones, pero la liga dentro de un marco de confianza.

- Responsabilidad: si los seguidores quieren llegar a tener algún tipo de poder, deben aceptar la responsabilidad por su rol y por los roles de sus líderes. Solo aceptando esta doble responsabilidad es como ellos finalmente aceptarán la responsabilidad por su organización y por las personas a las que sirven. Sin embargo, Chaleff 
(1997) estableció tres razones que los seguidores deben entender para asumir esta responsabilidad: primera, los seguidores deben entender su poder y cómo usarlo. Los seguidores tienen más poder de lo que ellos normalmente piensan. Ellos necesitan entender la fuente de su poder, a quienes ellos sirven y qué herramientas tienen para llevar a cabo la misión del grupo. Segunda, los seguidores deben apreciar la importancia de los líderes y sopesar las críticas como contribuciones que ellos hacen a los esfuerzos de los seguidores. Estos deben aprender a minimizar fuerzas y a crear un clima en el cual las fuerzas de los líderes sean magnificadas, así el líder podrá servir mejor al propósito común. Y tercera, los seguidores deben entender la seducción y las trampas que tiene el poder de liderazgo.

Los seguidores efectivos asumen esta responsabilidad de aprender las normas del sistema en el cual ellos operan. Así mismo, la actitud del seguidor frente a las normas es de suma importancia. Esto puede afectar las relaciones con el líder y sus respuestas al líder cuando se den órdenes inadecuadas.

- Procesos de comunicación: una organización apropiada es necesaria para llevar a cabo el propósito común y para dar respaldo al líder. Entonces, los seguidores efectivos son los que ayudan a los líderes a clarificar la visión y los objetivos, y a desarrollar la organización que el grupo necesita para llevarlos a cabo. Las estructuras organizativas más importantes y los procesos son aquellos que conllevan comunicación. Ellos dicen cómo todos los otros procesos organizacionales están funcionando. Para darle un buen servicio al líder, los seguidores deben ayudarlo a detectar las deficiencias en la comunicación y a diseñar la correcta combinación de los medios para satisfacer las necesidades de la organización.

Según Chaleff (1997), el diseño de los procesos de comunicación debería sensibilizarse con las necesidades múltiples, incluyendo la necesidad del líder de comunicar su visión directamente a todos los niveles de la organización y a sus responsables del legado, que los portadores del legado estén en capacidad de comunicar sus puntos de vista a los líderes, que la información se mueva fácilmente hacia arriba, hacia abajo, hacia los lados, dentro y fuera de la organización, de tal manera que ninguna parte del grupo o su ambiente quede desconectado, que la correcta combinación de media y tecnologías facilite la comunicación en un conjunto de situaciones, comunicaciones que se orienten a la creatividad, toma de decisión compartida, coordinación, implementación y evaluación.

Para esta investigación, el seguidor será entendido como: "una persona que reconoce el líder 1 como una continua fuente 
de orientación e inspiración, sin importar si hay alguna relación formal" (Yukl, 1998, p.6).

\section{SEGUIDORES Y SU RELACIÓN CON EL LÍDER}

La idea de que las características de los seguidores influyen en el impacto del liderazgo no es algo nuevo en el estudio de esta temática (Dvir, 1998). Castro (2007) señala que:

los miembros de un grupo de trabajo desarrollan, a través de procesos de socialización y experiencias pasadas con líderes, una serie de teorías implícitas acerca del liderazgo (TILs) (sic). Éstas consisten en un conjunto de presunciones personales acerca de atributos y habilidades que caracterizan a un líder ideal. Más específicamente, las TILs (sic) presuponen una serie de estructuras cognitivas o esquemas que especifican una serie de rasgos y conductas que los seguidores esperan de sus líderes(2007, p. 8).

De esta forma es posible identificar cómo esa relación y percepción del seguidor con el líder termina afectando los rasgos de este último. Razón por la cual, Castro (2008) sostiene que

(...) esencia del liderazgo reside más en el seguidor que en las características individuales del propio líder. De esta manera, Lord, Foti y De Vader (1984) argumentan que las diferentes percepciones que los seguidores sostienen acerca de sus superiores, conforman una serie de categorías cognitivas (o esquemas) jerarquizadas, cada una de las cuales está representada por una serie de prototipos (Castro, 2008, p.8).

En este caso específico, de acuerdo con el enfoque de Lord, Forti y De Vader (1984), la percepción del seguidor se convierte en elemento determinante de los rasgos del líder, y no solo como se ha planteado en los enfoques tradicionales del liderazgo, donde el líder establece en cierto grado los rasgos y el desempeño de sus seguidores, según los procesos de interacción que establece con ellos. El liderazgo pasa a ser entonces un proceso bilateral de relación entre líder y seguidor, donde este último fija ciertas percepciones como punto de partida para esperar la aparición de ciertos rasgos y conductas del líder. Razón por la que también se podría pensar que el desempeño de los seguidores pasa a ser resultado de la aparición de las características especiales en el líder, con las cuales puede mejorar sus procesos de influencia en los seguidores.

Por lo tanto, los miembros de un grupo de trabajo desarrollan, a través de procesos de socialización y experiencias pasadas con líderes, una serie de teorías implícitas de liderazgo (TIL), queconsisten en un conjunto de atribuciones personales acerca de las cualidades y habilidades que caracterizan a un líder ideal y las cuales finalmente se ocupan de la asignación del liderazgo (Kenney, Schartz-Kenney \& 
Blascovich, 1996). Asimismo, la teoría de intercambio líder-miembro (LMX) se basa en la idea fundamental de los intercambios dados entre seguidores y líderes (Gerstner \& Day, 1997; Graen \& UhlBien, 1995; Liden, Sparrowe \& Wayne, 1997). Esta teoría sostiene que la relación entre líderes y seguidores se media por la transacción realizada ente ellos. Algunos estudios han mostrado que esta teoría suele elegir cierto grupo de subordinados o seguidores más compatibles con el líder, ya sea por género u otra característica (Molero \& Morales, 2011). En general, la transacción que se hace entre líder y seguidor se da por la diferenciación o no de la relación y las características propias de ella (Liden, Erdogan, Wayne \& Sparrowe, 2006). Henderson, Liden, Glibkowski y Chaudhry (2009) indican como el tratamiento diferencial de los líderes de varios subordinados en un grupo de trabajo influye en la actividad dentro del grupo en general.

En este sentido, "un líder será considerado como tal si las características o conductas que de él perciben sus seguidores coinciden con los prototipos internalizados por ellos (es decir, si existe coincidencia entre el prototipo ideal y la percepción del líder real" (Castro, 2008, p.8).

Alves (2000) presenta las características de los liderados desde el modelo propuesto por Chelladurai, el cual prevé la influencia de las características de los liderados en el comportamiento del líder. Según el autor, su investigación demostró que los liderados difieren en su deseo y en su necesidad del liderazgo. La capacidad de asumir responsabilidades por parte de los seguidores no será un común denominador en todos, y por el contrario, estará determinada por las condiciones particulares de cada uno en relación con el líder.

Chaleff (1995) estableció el rol de los seguidores según diferentes niveles:

- En el más alto nivel, los seguidores sirven a aquellos para quienes la organización existe -susmiembros, los clientes, los parroquianos y las comunidades-frecuentemente llamados responsables del legado, debido a su compromiso con los resultados de las acciones del grupo.

- Por debajo de este primer nivel, y bastante funcional, los seguidores están al servicio de los responsables del legado y de sus líderes, sin que se dé un conflicto de intereses.

- Más abajo, los seguidores sirven a sus líderes y a ellos mismos, pero no a los portadores del legado. Aun cuando los seguidores pueden ser recompensados por esto a corto plazo, ellos están sembrando la semilla del fracaso.

- Por último, en el nivel más bajo, los seguidores sirven a sus líderes pero al mismo tiempo permiten que estos le hagan daño a la organización y a los responsables del legado por corrupción, además, los seguidores participan ellos mismos de esta corrupción. 
Según lo anterior, si los seguidores se sirven solo a ellos mismos y no a los líderes y a los responsables del legado, los seguidores no son leales. Por consiguiente, los seguidores necesitan estar muy al corriente acerca de su rol dentro del proceso de la organización.

Adicional a ello, Davis y Newstrom (2000) establecen algunas conductas que deben tener los seguidores para apoyar a los líderes y ser seguidores eficaces:

- No competir con el líder por atraer los reflectores

- Ser leales y brindar apoyo como miembros del equipo

- No estar automáticamente de acuerdo con todo

- Actuar como "abogados del diablo" planteando preguntas profundas

- Confrontar constructivamente las ideas, valores y acciones del líder

- Prever y prevenir posibles problemas

Para Northouse (2001), dentro del término liderar se encuentra la descripción de ejercer influencia sobre otros y generar visiones para el cambio, es decir, que implica una relación de influencia entre iguales y no incluye órdenes, por lo cual los seguidores tienen un rol de iguales frente a los líderes.

Bass (1985), por su parte, menciona que el líder es un agente que transforma a los seguidores haciéndolos más conscientes de la importancia y el valor de los resultados, llevándolos a transcender su propio autointerés, por el bien de la organización.
Como resultado, los seguidores sienten respeto y confianza hacia su líder y están motivados a hacer más de lo que ellos originalmente esperaban hacer.

Aunque el líder eficaz pueda en ocasiones señalar un destino específico que la gente se sienta obligada a buscar, es más probable que su eficacia resida, sobre todo, en su capacidad de poner en marcha el proceso de orientación (Weick, 2000, p. 107). Las relaciones entre líderes y seguidores determinan la efectividad de cada proceso. Es esta relación hacia arriba y hacia debajo de la organización la que construye programas, daña programas y hace o termina con las carreras.

Padilla y Quintana (2012) citando a Munchinsky (2006), plantean la posibilidad de estudiar los procesos de influencia no solo del líder hacia los seguidores sino en el sentido contrario, para determinar cómo las acciones de los seguidores afectan el liderazgo. Autores como Bennis, (1990) y Chaleff, $(1995,1997)$ afirman que el hecho de ser seguidores requiere respaldo leal y enérgico del trabajo del líder, así como la voluntad de retar las políticas del líder o sus comportamientos, si estos llegaren a ser nocivos al propósito común. Sin embargo, se sabe que dentro de una sociedad inclinada al liderazgo, muchos se incomodan con eso de ser seguidor, aun cuando ambas cosas son inseparables.

Por su parte, López, Villagómez y Cruz de Galindo (2003) afirman que:

El liderazgo es una fuente de poder 
de abajo hacia arriba; un poder que se entrega, como uno de los elementos importantes en el proceso de abdicación del seguidor: el líder se subroga en los afanes personales del seguidor y este a cambio le otorga el poder (p. 52).

Para este autor, la influencia ejercida en el liderazgo es de doble vía, no solo hacia abajo sino hacia arriba, dependiendo de los intereses y necesidades que los seguidores manifiestan. Los líderes, al igual que los seguidores, no son receptores pasivos de la influencia del seguidor. Ellos tienen sus propias diferencias individuales, preferencias y percepciones que pueden amplificar o atenuar la influencia de los seguidores (Oc \& Bashshur, 2013).

Por su parte, Barnard (2003) habla de la autoridad que es aceptada o no por el seguidor. Este autor manifiesta que cuando el poder va en una dirección, de líder a seguidor, son finalmente los seguidores quienes deciden si la autoridad es legítima y si debe o no aceptarse. En este sentido, en el ejercicio del liderazgo se estable el reconocimiento del poder, a partir de la aceptación o no del seguidor.

Muchos escritores (Graen \& Uhl-Bien, 1995; Hollander, 1993; Jermier, 1993; Klein \& House, 1995; Yukl \& VanFleet, 1992) están de acuerdo que en el liderazgo la relación se produce conjuntamente por líderes y seguidores. Los líderes y los seguidores desempeñan un papel activo en la conformación de sus relaciones mutuas, y hay un primer plano en la confor- mación de los resultados organizacionales (Dvir, 1998; Graen \& Uhl-Bien, 1995). Kelley (1992) afirma que para muchos, el rol de seguidor es una elección consiente. Los mejores seguidores, dice, son los que se comprometen activamente con sus líderes y su ambiente y que se manifiestan de pensamiento independiente y crítico.

Por lo tanto, la esencia del liderazgo reside más en el seguidor que en las características individuales del propio líder. Una persona surge como líder si el grupo le atribuye características propias del liderazgo relacionadascon los seguidores (Lord \& Maher, 1991).

Chaleff (1995) afirmó que le valor de un seguidor se mide por el modo como este ayuda al líder y a la organización a perseguir el propósito común dentro del contexto de sus valores. El afirma que ciertas características ayudan en esta labor:

- Los seguidores eficientes son cooperadores y colaboradores, cualidades esenciales a todo progreso humano.

- Los seguidores confiables son aquellos que integran las necesidades de su ego con las responsabilidades de la comunidad que sirven, en vez de competir con su líder.

- Los seguidores equilibrados son menos propensos a caer en las trampas que asechan a los líderes con fuertes egos y pueden servir de guías en estas trampas.

- Los seguidores cuidadosos perciben las necesidades tanto de los líderes como de otros miembros del grupo y tratan de establecer un puente entre ellos. 
Las personas conservan sus valores como seguidores en la medida en que permanecen fieles a aquellos a quienes sirve la organización y en la medida en que sean valerosos para hacer esto. Si se inclinan a la voluntad del líder cuando este entra en conflicto con los intereses de los responsables del legado, o si ellos se inclinan a la voluntad de los responsables del legado cuando estos entran en conflicto con valores más importantes, sus valores se van a encontrar fuertemente disminuidos.

Autores como Lord, Brown y Feiberg (1999) y Shamir (2007) establecieronque una fuente importante de variación son las diferencias en los autoconceptos de los seguidores. Estos son poderosos determinantes del comportamiento del seguidor y de las reacciones que estos tengan hacia sus líderes. Por otra parte, los líderes pueden influenciar poderosamente los autoconceptos de sus subordinados y en consecuencia influenciar el comportamiento del seguidor y otros procesos sociales.

Esta es una razón para pensar que el liderazgo es un proceso que no depende solo del líder sino también de sus seguidores. Chaleff (1995) sostenía que pensar en líderes sin seguidores es como pensar en profesores sin estudiantes. Ambas cosas son imposibles. Ellos son dos caras del mismo proceso, dos partes del mismo todo. Líderes y seguidores forman un círculo de acción alrededor de un propósito común.

Este enfoque centrado en el seguidor le asigna un papel activo dentro del lide- razgo, sin embargo, las preferencias o actitudes (por influencia de sus rasgos y la excitación emocional) de los seguidores pueden, de forma pasiva, o incluso activa, frenar los procesos de liderazgo, según lo plantean algunos autores como Ehrhart y Klein (2001); Grant, Gino, y Hofmann (2011); y Kark, Shamir y Chen, (2003).

PAPEL DE LOS SEGUIDORES EN CADA UNA DE LAS TEORÍAS DEL LIDERAZGO

Gil, Alcover, Rico y Sánchez (2011) citan a Weick en su artículo y mencionan que los nuevos escenarios en los que intervienen los líderes hoy en día se caracterizan por dar prioridad al movimiento continuo frente al distanciamiento y la reflexión, por el desplazamiento de las decisiones a los expertos en lugar de a las personas autorizadas a tomarlas por sus cargos, por una mayor improvisación y una menor rutina, por ser más importante la actualización y la razonabilidad que la predicción y la precisión, y por ser más recomendable la humildad en lugar de la arrogancia. Ahora bien, los seguidores en estos nuevos escenarios pasan a ser de agentes de reflexión donde esperan a que los líderes den coherencia y sentido a lo que hacen. En este caso, explica Weick (2000), que la coherencia es mantenerse en contacto con el contexto y no dar respuestas genéricas o correctas a la hora de tomar decisiones.

La mayoría de las teorías del liderazgo se centra enlas conductas del líder, o la estructuración de las tareas, la conside- 
ración y el apoyo de los seguidores, y la inclusión de los seguidores en la toma de decisiones, no obstante, se concibe el papel de seguidores desde el punto de vista de su susceptibilidad a ciertos comportamientos o estilos líder (Howell \& Shamir, 2005).

La visión habitual de liderazgo enmarca a los seguidores como receptores pasivos de las características del líder, como rasgos, habilidades y comportamientos, y se limitaa examinar el flujo de la influencia de los líderes de seguidores (Graen \& Uhl-Bien,1995; Hollander, 1980, 1992). Por lo tanto, a continuación se presenta el rol específico que tiene el seguidor dentro de cada una de las teorías del liderazgo:

\section{Teoría de los rasgos y el comportamiento}

Hemos enmarcado el rol del seguidor juntando estas dos teorías, ya que el seguidor visto desde este punto ejerce su influencia a partir de la percepción que tiene de los rasgos y el comportamiento del líder. Dentro de esta teoría, Howell y Shamir (2005) afirman que los seguidores también desempeñan un papel más activo en la construcción de la relación de liderazgo, el empoderamiento del líder e influencia su comportamiento. Estos dos autores se centran especialmente en las descripciones de seguidores bajo la idea de seguir a líderes carismáticos, a los cuales se les atribuyen rasgos y comportamientos de líderes.

Estos autores plantean que la relación de carisma en esta teoría se da directa- mente por el seguidor. En concreto, las características personales de los líderes carismáticos que contribuyen a la formación de una relación carismática incluyen autoconfianza, necesidad de influencia, convicción moral y asertividad prosocial asignada directamente por el seguidor (Bass, 1985; Conger \& Kanungo, 1998; House et al., 1991).

Howell y Shamir (2005) también plantean que dependiendo de los conceptos de sí mismos, los seguidores pueden desarrollar dos tipos de relación con el líder basado en sus rasgos:personalizada o socializada, las cuales se basan en los argumentos de Howell (1988), House y Howell (1992), Kark y Shamir (2002), Shamir et al. (1993) y Weierter (1997). La relación personalizada que plantean estos autoresse caracteriza por la atribución de cualidades deseables al líder, una definición de sí mismo en términos de la relación con el líder, y el deseo de llegar a ser como el líder.

En la relación socializada, los seguidores tienen un claro sentido de sí mismos y un conjunto claro de valores. La relación carismática les proporciona un medio para la expresión de sus valores importantes en el marco de una acción colectiva. Seguidores de este tipo derivan su sentido de la orientación y la expresión, no de identificación personal con el líder. La relación de los seguidores tiene restricciones sobre la influencia del líder, además, ellos desempeñanun papel activo en la determinación de los valores expresados por el líder, son menos dependientes del 
líder, y son menos susceptibles de manipulación por el líder (Howell \& Shamir, 2005).

Ehrhart y Klein (2001) sostienen que un líder al que se le examina en relación con sus rasgos y comportamiento, como lo es el líder carismático, comunica expectativas altas de desempeño a los seguidores, muestra seguridad en alcanzar sus metas, toma riesgos calculados para romper el statu quo, por lo tanto, los seguidores que están orientados al logro, que tienen alta autoestima y disfrutan tomando riesgos, influyen directamente en este líder. Según lo plantean estos autores, el rol de este seguidor en esta teoría está alineado directamente a ciertas características del seguidor que vincula directamente con el líder.

Conger, Kanungo, Menon y Mathur (1997) se refieren específicamente al liderazgo carismático, donde el papel del seguidor está dado en una atribución basada en percepciones sobre la conducta de su líder. El comportamiento observado del líder es interpretado por los seguidores como expresiones de su carisma en el mismo sentido que los comportamientos de un líder reflejan las orientaciones que este posee.

\section{Teoría de la contingencia}

Esta perspectiva limitada acerca del papel pasivo del seguidor comenzó a cambiar radicalmente desde la teoría de la contingencia (Oc \& Bashshur, 2013). En muchas de estas teorías, los efectos del seguidor se modelan de forma explícita, como lo hacen Fiedler (1967) y Evans (1970). En la teoría de contingencia de Fiedler, la relación entre el estilo de liderazgo y la efectividad del líder se basa en el contexto, en particular la calidad de las relaciones líder-seguidor. Fiedler (1967) incluye la lealtad de los seguidores, apoyo y cooperación con el líder, como un factor determinante de la situación de las personas orientadas a las relaciones o a las tareas.

Otras teorías de contingencia, como las de Hersey y Blanchard (1969), sugieren que los líderes deben encontrar un equilibrio entre las tareas y los comportamientos orientados a las relaciones en función de la confianza y la habilidad conjuntas de sus seguidores. Afirmación que más adelante sustentan Evans (1970), House (1971) y House y Mitchell (1974). Además, estos autores sostienen que las características del seguidor son factores clave para dar forma a la efectividad del líder, encontrando ahí el papel del seguidor. Asimismo, Hersey y Blanchard (1977) se refieren al seguidor en su nivel de madurez, habilidades y confianza, como factores que influyen en la idoneidad de diferentes estilos de liderazgo.

Kerr y Jermier (1978), y Vroom y Yetton (1973) incluyen varias características en el modelo de estilos de toma de decisiones, y el liderazgo que incluye la experiencia, capacidad, formación y orientación profesional como factores que pueden negar la necesidad de liderazgo o contener su impacto en diversos resultados. 


\section{Teorías emergentes}

Desde la teoría del liderazgo transaccional y transformacional, donde el primero se caracteriza por el intercambio entre líderes y sus seguidores, y el segundo consiste en comprometer a otros y crear una conexión que da lugar a un nivel de motivación y moralidad en ambos, el líder y el seguidor. En este sentido, la influencia de los líderes transformacionales se genera de la aceptación personal de ciertos valores de sus seguidores, y estos proporcionan una pauta para la toma de decisiones y la conducta, por lo que los seguidores que comparten los valores del líder no dependen de sus órdenes (Hellriegel, Slocum \& Woodman, 1999, p. 345).

Burns (1978) afirma que la transformación de liderazgo es un proceso de influencia mutua entre el líder y los seguidores, pero su análisis de este tipo de liderazgo, como el de Bass (1985), se centra principalmente en el líder. Este mismo autor afirmó que el liderazgo transformacional propone que el líder establezca los valores más altos, el propósito, o forma de autorrealización, y que a partir de eso la relación entre el líder y el seguidor una esos propósitos y los lleve a un nivel más alto.

Burns (1978) y Bass (1985) plantean que el liderazgo bajo esta teoría ha sido generalmente entendido como un proceso transaccional o de intercambio, definiendo la relación entre líder y seguidor como una transacción de intereses en la cual tanto líder como seguidor obtienen sus propios beneficios. En el texto de Burns (1978) se hace una fuerte crítica de este líder en virtud de su relación con el seguidor, ya que antepone sus propias necesidades a las de sus seguidores y lo convierte en un inmaduro. Por lo tanto, bajo el criterio de este autor, en esta teoría el rol del seguidor tiene mayor relevancia que la del líder y es el que determina su comportamiento. Bass (1985) también señala dentro de otro texto que el líder transformacional inspira a los seguidores a hacer más de lo esperado.

Bass y Avolio (1994) señalan que el liderazgo transformacional se considera como una expansión del liderazgo transaccional y surgen con la intención de enriquecer y mejorar la relación líderseguidor. Los seguidores de los líderes transformacionales están más conscientes de la importancia de supuestos y de un desempeño de alto nivel, están conscientes de sus necesidades personales de crecimiento, desarrollo y realización y trabajan también para su beneficio personal (Jones \& George, 2010).

Los seguidores de los líderes transformacionales experimentan una creencia total e identificación con los líderes y su misión. Por lo tanto, los líderes transformacionales son vistos como serviciales y amables (Krishnan, 2004). El liderazgo transformacional implica una interacción fluida entre los dos actores, líder-seguidor, el primero intentando motivar, influir e incrementar la madurez de los seguidores para que traten de ir más allá de sus propios intereses, orientándolos a 
un pensamiento de grupo, hacia la organización y de la sociedad.

\section{CONCLUSIONES}

En el desarrollo de este artículo se identifica que tanto el líder como el seguidor son importantes en el proceso de liderazgo. Aun cuando se otorgue más importancia al líder, este necesita a los seguidores para desarrollar un buen trabajo. Además, es posible que una persona se encuentre comprometida en ambas situaciones o roles. Sin embargo, la persona puede ser un buen seguidor sin aspirar a un rol de líder.

Adicionalmente, ambos, líderes y seguidores, necesitan entender y respetar la actividad de ser seguidor. Los líderes solo deben esperar lo mejor de los seguidores cuando la decisión de ser seguidor se hace explícita y a su vez modelan este comportamiento cuando es apropiado. Asimismo, al modelar buenas relaciones entre seguidores y líderes, los seguidores necesitan influenciar a los líderes. Si no pueden influenciar a sus líderes, ellos pueden transformar una relación pobre en una oportunidad de aprendizaje rica, siempre y cuando aprendan lo que motiva a los seguidores y se comprometan a no repetir estos errores. Para amparar efectivamente a un líder, los seguidores necesitan crear su respaldo para ejecutar esa parte de la misión con la que ellos están comprometidos. Entonces el proceso de liderazgo necesita y exige a ambos, tanto líderes como seguidores, pues son inseparables.
A los seguidores durante muchos años se les otorgó un rol pasivo dentro del proceso de liderazgo, sin embargo, la mayoría de las investigaciones señalan que el seguidor tiene un papel activo, el cual empieza a ser descubierto desde la teoría convergerte.

La teoría de los rasgos y el comportamiento tienen una postura dada por la percepción de los seguidores para identificar a su líder y la alineación que tienen con esto. Bajo esta teoría, el líder solo existe si es percibido por los seguidores y si este posee características individuales y se comporta tal como lo aceptan los seguidores.

La teoría de la contingencia, por su parte, formula un papel del seguidor mucho más activo que en la teoría anterior, ya que, como lo plantea la mayoría de los actores, el líder actúa de acuerdo con su contexto y las exigencias del seguidor. Este líder siempre está en función de lo que le está brindando la situación para actuar adecuadamente. Esta teoría aplicada a las organizaciones sostiene que el líder puede mejorar la eficacia de la organización si su contexto y los seguidores brindan la oportunidad de que se escoja un estilo de liderazgo adecuado.

La teoría emergente presenta un papel del seguidor bajo el cual se está en constante transacción con este, es decir, el seguidor siempre le está ofreciendo al líder lo que quiere y bajo esas circunstancias, el líder puede transar con sus seguidores para ga- 
rantizar la efectividad en el cumplimiento del logro.

La revisión de la literatura realizada en este artículo nos abre las puertas a la visión que podemos tener de los seguidores y el paradigma bajo el cual los vamos a evaluar para determinar la influencia que tienen en el líder. Sin embargo, la revisión de la literatura se limita al caso teórico del ejercicio del seguidor, por lo que sería interesante más adelante evaluar de manera empírica y con el uso de cuestionarios aplicables, el papel del seguidor en el líder bajo un contexto determinado.

\section{REFERENCIAS}

1. Alves, J. (2000). Liderazgo y clima organizacional. Revista de Psicología del Deporte, 9(1-2), 123-133.

2. Antonakis, J., Cianciolo, A. \& Sternberg, R. (2004).The nature of leadership.Thousand Oaks, CA: Sage.

3. Barnard, C. I. (2003). Organization and management: Selected papers (Vol. 7). Cambridge: Psychology Press.

4. Bass, B. M (1981). Stogdill's handbook of leadership: a survey of theory and research. New York: Free Press.

5. Bass, B. M. (1985). Leadership and performance beyond expectations. New York: Free Press.

6. Bass, B.M. (1990). Bass and Stodgill's handbook of leadership, (third ed.). New York: Free Press.

7. Bass, B.M. \&Avolio, B.J. (1994).Improving organizational effectiveness through transformational leadership, Thousand Oaks, C.A: Sage Publications.

8. Bennis, W. (1990). Cómo llegar a ser líder. Bogotá: Norma.

9. Blake, R. \& Mouton, J. (1964).The managerial grid: key orientations for achieving production through people. Houston, Texas: Gulf Publishing Company.

10. Burns, J. M. (1978). Leadership. New York: Harper and Row.

11. Can, A., \& Aktaş, M. (2012). Cultural values and followership style preferences. Procedia Social and Behavioral Sciences, 41(0), 84-91. Retrieved from http://dx.doi.org/10.1016/j. sbspro.2012.04.012 
12. Carsten, M. K., Uhl-Bien, M., West, B. J., Patera, J. L. \& McGregor, R. (2010). Exploring social constructions of followership: a qualitative study.The Leadership Quarterly, 21(3), 543-562.Retrieved from http://dx.doi.org/10.1016/j.leaqua.2010.03.015

13. Castro, A. (2008). Teorías implícitas del liderazgo, calidad de larelación entre líder, seguidor y satisfacción. Anuario de Psicología, 39(3), 333-350.

14. Castro, A. (2007). Teorías implícitas del liderazgo y calidad de la relación entre líder y seguidor. Boletín de psicología, (89), 7-28.

15. Chaleff, I. (1995).The courageous follower: standing up to and for our leaders. San Francisco: Berrert - Koehler Publishers.

16. Chaleff, I. (1997). Learn the art of followership. Government executive, 29(2), 51.

17. Collinson, D. (2006). Rethinking followership: a post-structuralist analysis of follower identities.The Leadership Quarterly, 17(2), 179-189. Retrieved from http://dx.doi. org/10.1016/j.leaqua.2005.12.005

18. Conger, J.A. \& Kanungo, R.N. (1987). Toward a behavioral theory of charismatic leadership in organizational settings. Academy of Management Review, 12, 637-647.

19. Conger, J.A. \& Kanungo, R.N. (1998). Charismatic Leadership in Organizations. Thousand Oaks, CA: Sage Publishers.

20. Conger, J. A., Kanungo, R. \& Menon, A. (2000).Charismatic leadership and follower effects. Journal Organizational Behavior,21(7) 747-767.

21. Conger, J.A., Kanungo, R.N., Menon, S.T. \& Mathur, P. (1997). Measuring charisma: dimensionality and validity of the Conge Kanungo scale of charismatic leadership. Canadian Journal of Administrative Sciences, 14(3), 290-302.

22. Davidovitz, R., Mikulincer, M., Shaver, P., Izak, R. \& Popper, M. (2007). Leaders as attachment figures: leaders' attachment orientations predict leadership-related mental representations and followers' performance and mental Health. Journal of Personality and social Psychology, 93(4), 632-650.

23. Davis, K. \&Newstrom, J.W. (2000). Comportamiento humano en el trabajo. México D.F.: McGraw Hill.

24. Dvir, T. (1998). Impact of transformational leadership training on follower development and performance: A field experiment. Unpublished doctoral dissertation, Faculty of Management, Tel-Aviv University, Israel.

25. Ehrhart, M. G. \& Klein, K. J. (2001). Predicting followers' preferences for charismatic leadership: The influence of follower values and personality. The Leadership Quarterly, 12(2), 153-179. 
26. Erez, A., Misangyi, V., Johnson, D., Lepine, M. \& Halverson, K. (2008). Stirring the hearts of followers: charismatic leadership as the transferal of affect. Journal of applied psychology. 93(3), 602-615.

27. Evans, M. G. (1970). Leadership and motivation: a core concept. Academy of Management Journal, 13(1), 91-102.

28. Evkall, G. \& Ryhammar, L. (1997). Leadership style, social climate and organizational outcomes: A study of a Swedish University College. Creat. Innov. Manag. 7, 126-130.

29. Fayol, H. (1986). Administración industrial y general ( $3^{\mathrm{a}}$ ed.). Barcelona: Orbis.

30. Felfe, J. \& Schyns, B. (2006). Personality and the perception of transformational leadership: the impact of extraversion, neuroticism, personal need for structure, and occupational efficacy. Journal of Applied Social Psychology, 36(3), 708-739.

31. Fiedler, F. E. (1967). A theory of leadership effectiveness. New York: McGraw Hill.

32. Fleishman, E. A., Mumford, M. D., Zaccaro, S. J., Levin, K. Y., Korotkin, A. L. \& Hein, M.B. (1991). Taxonomic efforts in the description of leader behavior: A synthesis and functional interpretation. Leadership Quarterly, 2, 245-287.

33. Gerstner, C. R. \& Day, D. V. (1997). Meta-analytic review of leader-member exchange theory: correlates and construct issues. Journal of applied psychology, 82(6), 827.

34. Gil, F., Alcover, C., Rico, R. \& Sánchez-Manzanares, M. (2011, enero-abril). Nuevas formas de liderazgo en equipos de trabajo. Papeles del Psicólogo, 38-47.

35. Graen, G. B. \& Uhl-Bien, M. (1995). Relationship-based approach to leadership: Development of leader-member exchange (LMX) theory over 25 years: Applying a multi level multi-domain perspective. Leadership Quarterly, 6, 219-247.

36. Grant, A. M., Gino, F. \& Hofmann, D. A. (2011). Reversing the extraverted leadership advantage: The role of employee proactivity. Academy of Management Journal,54(3), 528-550.

37. Guillen, M. (2006). Ética en las organizaciones: construyendo confianza. Madrid: Pearson Educación.

38. Hellriegel, D., Slocum, J.W. Jr. \& Woodman, R.W. (1999). Comportamiento Organizacional (8 ed.). México: Thomson.

39. Henderson, D. J., Liden, R. C., Glibkowski, B. C. \& Chaudhry, A. (2009). LMX differentiation: A multilevel review and examination of its antecedents and outcomes. The leadership quarterly, 20(4), 517-534. 
40. Hersey, P. \& Blanchard, K.H. (1969).Management of organizational behavior: Utilizing human resources. Englewoods Cliffs, NJ: Prentice-Hall.

41. Hersey, P. \& Blanchard, K.H. (1977). Management of organization behavior: utilizing human resources (3rd ed.). Englewood Cliffs, NJ: Prentice-Hall.

42. Hersey, P. \& Blanchard, K.H. (1988).Management of organizational behavior: Utilizing human resources. (5th ed.). Englewood Cliffs, NJ: Prentice-Hall.

43. Hollander, E. P. (1980). Leadership and social exchange processes. US: Springer.

44. Hollander, E. P. (1992). Leadership, followership, self, and others. The Leadership Quarterly, 3(1), 43-54.

45. Hollander, E. P. (1993). Legitimacy, power and influence: A perspective on relational features of leadership. In M. M. Chemersy \& R. Ayman (Eds.), Leadership theory and research: Perspectives and directions(29-48). San Diego: AcademicPress.

46. Hoption, C., Christie, A. \& Barling, J. (2012).Submitting to the follower label: Followership, positive affect, and extra-role behaviors.Zeitschrift für Psychologie/Journal of Psychology, 220(4), 221-230.

47. House, R. J. (1971, september). A path-goal theory of leader effectiveness. Administrative Science Quarterly, 16(5), 321-339.

48. House, R. J., Spangler, W. D. \& Woycke, J. (1991). Personality and charisma in the U.S. presidency: A psychological theory of leadership effectiveness. Administrative Science Quarterly, 36, 374-396.

49. House, R. J. \& Howell, J. M. (1992). Personality and charismatic leadership. Leadership Quarterly, 3, 81-108.

50. House, R. J. \& Mitchell, T. R. (1974).Path-goal theory of leadership. Journal of Contemporary Business, 3, 81-97.

51. Howell, J. M. (1988). Two faces of charisma: Socialized and personalized leadership in organizations. In J. A. Conger, R. N. Kanungo and Associates (eds.). Charismatic leadership: The elusive factor in organizational effectiveness(213-236). San Francisco: Jossey-Bass.

52. Howell, J. M. \& Shamir, B. (2005). The role of followers in the charismatic leadership process: Relationships and their consequences. Academy of Management Review, 30(1), 96-112.

53. Howell, J. \& Hall-Merenda, K. (1999). The ties that bind: the impact of leader-member exchange, transformational and transactional leadership, and distance on predicting follower performance. Journal of Applied Psychology, 84(5), 680-694. 
54. Jermier, J. M. (1993). Introduction: Charismatic leadership: Neo-Weberian perspectives. Leadership Quarterly, 4, 217-234.

55. Jones, G. \& George, J. (2010). Administracion contemporánea. México: McGraw Hill.

56. Kahai, S. \& Sosik, J. (1997). Effects of leadership style and follower's cultural orientation on performance in group and individual task conditions. Personnel Psychol, 50, 121-147.

57. Kark, R., Shamir, B. \& Chen, G. (2003). Thetwo faces of transformationalleadership: Empowerment and dependency. Journal of AppliedPsychology, 88, 246-255.

58. Kark, R. \& Shamir, B. (2002). The dual effect of transformational leadership: Priming relational and collective selves and further effects on followers. In B. J. Avolio\& F. J. Yammarino (eds.). Transformational and charismatic leadership: The road ahead (67-91). Oxford: Elsevier Science.

59. Kelley, R.E. (1988). In praise of followers. Harvard Business Review, 66(6), 142-148.

60. Kelley, R. E. (1992). The power of followership. New York: Doubleday Currency.

61. Kelley, R. E. (1992). Líderes y seguidores: Cooperación mutua en beneficio de la empresa. Madrid: McGraw-Hill Interamericana de España.

62. Kenney, R.A., Schwartz-Kenney, B.M. \&Blascovich,J. (1996). Implicit leadership theories: defining leaders described as worthy of influence. Personality and Social Psychology Bulletin, 22, 1128-1142.

63. Kerr, S. \& Jermier, J. M. (1978). Substitutes for leadership: their meaning and measurement. Organizational Behavior and Human Performance, 22, 375-403.

64. Klein, K. J. \& House, R. J. (1995). On fire: Charismatic leadership and levels of analysis. Leadership Quarterly, 6,183-198.

65. Kohles, J. C., Bligh, M. C. \& Carsten, M. K. (2012). A follower-centric approach to the vision integration process. The Leadership Quarterly, 23(3), 476-487. Retrieved from http://dx.doi.org/10.1016/j.leaqua.2011.12.002

66. Krishnan, V. R. (2004).Impact of transformational leadership on followers' influence strategies. LeadershipOrganizationDevelopment Journal, 25(1), p. 58-72.

67. Lee, J., Almaza, B., Jang, S., Nelson, D. \& Ghiselli, R. (2013). Does transformational leadership style influence employees' attitudes toward food safety practices? International Journal of Hospitality Management, 33, 282-293.

68. Lewin, K. (1951). Field theory in social science.Nueva York: Harper.

69. Liden, R. C., Sparrowe, R. T. \& Wayne, S. J. (1997). Leader-member exchange theory: The past and potential for the future. Research in personnel and human resources management, 15, 47-120. 
70. Liden, R. C., Erdogan, B., Wayne, S. J. \& Sparrowe, R. T. (2006). Leader member exchange, differentiation, and task interdependence: implications for individual and group performance. Journal of Organizational Behavior, 27(6), 723-746.

71. Likert, R. (1961). New patterns of management. New York: McGraw-Hill.

72. López, D.L., Villagómez, A. \& Cruz de Galindo, L. (2003). Líderes, directores y seguidores en la actividad laboral. Hospitalidad ESDAI, (3), 49-78.

73. Lord, R. G. \& Maher, K. J. (1991). Leadership and information processing. Londres: Routledge.

74. Lord, R., Foti, R. \& De Vader, C. (1984). A test of leadership categorization theory: Internal structure, information processing andleadership perceptions. Organizational Behaviour and Human Performance, 34, 343-378.

75. Lord, R., Brown, D. \& Freiberg, S. (1999).Understanding the dynamics of leadership: the role of follower self-concepts in de leader/follower relationship. Organizational Behavior and Human Decision Processes, 78.

76. Lussier, R.N. \& Achua, C.F. (2002). Liderazgo. Teoría, aplicación y desarrollo de habilidades, (2a. ed.). México: Thomson Learning.

77. Meindl, J. R. (1995). The romance of leadership as a follower-centric theory: A social constructionist approach. The Leadership Quarterly, 6, 329-341.

78. Miller, R. Butler, J. \& Consentino, C. (2003). Followership effectiveness: an extension of Fiedler's contingency model. Leadership and Organization Development Journal, 25 (4), 7.

79. Molero, F. \& Morales, J. F. (2011). Liderazgo: hecho y ficción. Visiones actuales. Madrid: Alianza.

80. Nader, M. (2012). Teorías implícitas del liderazgo, LMX y bienestar laboral: generalización de un modelo teórico. Revista de Psicología, 28(2), 227-258.

81. Northouse, P. (2001). Leadership Theory and Practice. (2th Ed.). Thousand Oaks, CA: Sage Publications.

82. Oc, B. \& Bashshur, M. R. (2013). Followership, leadership and social influence. The Leadership Quarterly, 24(6), 919-934.

83. Padilla, S. \& Quintana, A. (2012). Impacto del liderazgo en las actitudes de los colaboradores. Anáhuac Journal, 12(2),51-72.

84. Palomino, J., Espinoza, J. \& Aguilar, M. (2013). Influencia del comportamiento del líder en el desarrollo de los recursos humanos. Global ConferenceonBusiness and Finance Proceedings, 8 (1). 
85. Palomino, P. R. (2009).Últimas tendencias en el estudio sobre liderazgo: revisión de la literatura. Documentos de Trabajo. Seminario Permanente de Ciencias Sociales, (5), 1-16.

86. Peterson, W. \& Avolio, H. (2012, June). The relationship between authentic leadership and follower job performance: The mediating role of follower positivity in extreme contexts. The Leadership Quarterly, 23(3), 502-516. doi.org/10.1016/j.leaqua.2011.12.004. Retrieved from http://www.sciencedirect.com/science/article/pii/S1048984311001998

87. Podsakoff, P., Mackenzie, S., Moorman, R. \& Fetter, R. (1990). Transformational leader behaviors and their effects on followers' trust in leader, satisfaction, and organizational citizenship behaviors. Leadership Quartely, 1 (2),107-142.

88. Rahman, S. (2001). Total quality management practices and business outcome: Evidence from small and medium enterprises in Western Australia. Total Quality Manag., 12, 201210 .

89. Robbins, S. P. (1999). Comportamiento organizacional, (8 ed.).México: Prentice Hall.

90. Shamir, B., House, R. \& Arthur, M.B. (1993). The motivational effects of charismatic leadership: a self-conceptbased theory. Organizational Science, 4(4), 577-594.

91. Shamir, B. (2007). From passive recipients to active co-producers: The roles of followers in the leadership process. In B. Shamir, R. Pillai, M. Bligh \& M. Uhl-Bien(Eds.), Followercentered perspectives on leadership: A tribute to J. R. Meindl. Stamford, CT: Information Age Publishing.

92. Sy, T. \& Choi, Y. (2013, November). Contagious leaders and followers: Exploring multistage mood contagion in a leader activation and member propagation (LAMP) model. Organizational Behavior and Human Decision Processes, 122(2), 127-140.

93. Sy, T. (2010). What do you think of followers? Examining the content, structure, and consequences of implicit followership theories. Organizational Behavior and Human Decision Processes, 113(2),73-84. Retrieved from http://dx.doi.org/10.1016/j. obhdp.2010.06.001

94. Theime, C. \& Treviño, E. (2012). Liderazgo en educación: al final solo el carisma importa. Espacio Abierto. Cuaderno Venezolano de Sociología, 21(1), 37-57.

95. Vroom, V. H. \& Yetton, P. W. (1973). Leadership and decision making. Pittsburgh: University of Pittsburgh Press.

96. Waldman, D. A., Ramírez, G. G., House, R. J. \& Puranam, P. (2001). Does leadership matter? CEO leadership attributes and profitability under conditions of perceived environmental uncertainty. Academy of management journal,44(1), 134-143. 
97. Weick, K. E. (2000). El liderazgo como legitimación de la duda. En W. Bennis, G. M. Spreitzer \& T. G. Cummimgs (Eds.), El futuro del liderazgo (pp. 104-115). Bilbao: Deusto.

98. Weierter, S. J. M. (1997). Who wants to play “follow the leader"? A theory of charismatic relationships based on routinized charisma and follower characteristics. Leadership Quarterly, 8, 171-194.

99. Yukl, G. (1998). Leadership in organizations (4th ed.). Upper Saddle River, NJ: PrenticeHall.

100. Yukl, G. \& Van Fleet, D. D. (1992). Theory and research on leadership in organizations. In M. D. Dunnette \& L. M. Hough (Eds.), Handbook of industrial and organizational psychology (2nd ed.),(147-197). Palo Alto, CA: Consulting Psychologists Press.

101. Zaccaro, S.J. (1995). Leader resources and the nature of organizational problems: commentary on cognitive resources and leadership. Appl. Psychol. 44. 